\title{
High-Frequency Ventilation: Rescue Me from Rescue
}

Rob Graham, R.R.T./N.R.C.P.

I dedicate this column to the late Dr. Andrew (Andy) Shennan, the founder of the perinatal program at Women's College Hospital (now at Sunnybrook Health Sciences Centre). To my teacher, my mentor and the man I owe my career as it is to, thank you. You have earned your place where there are no hospitals and no NICUs, where all the babies do is laugh and giggle and sleep.

I recently spent some time teaching new jet ventilator users. During the discussion after my talk, one of the physicians commented that "HFO is for rescue." Given that I have used high-frequency ventilation (HFV) as first intention for approximately 15 years, I had to take issue with the comment.

\section{"There is a saying in the information technology sector: "GIGO," meaning "garbage in, garbage out." One could use this expression when it comes to "rescue" ventilation modes; the worse a baby is before being offered the "rescue" therapy, the less successful the therapy is likely to be."}

There is a saying in the information technology sector: "GIGO," meaning "garbage in, garbage out." One could use this expression when it comes to "rescue" ventilation modes; the worse a baby is before being offered the "rescue" therapy, the less successful the therapy is likely to be. Indeed, it is a testament to the efficacy of HFV that it would be considered a rescue mode in the first place.

When the unit I practice in received its first second-generation oscillators (in our case, the Babylog® 8000 plus), I observed that most of our micro-preemies started on conventional ventilation (CV) ended up being oscillated. The thought came to mind "why not just start them on HFV"? That is what ended up happening over a few years of practice with the new machines, and we have never looked back; our chronic lung disease (CLD) rates improved and are to this day world-class. When we first started using the Bunnell LifePulse ${ }^{\circledR} 203$ ventilator, it too was used as a rescue device. Not surprisingly, a number of babies did not survive, to the point of us having a theme song for "the jet," sung to the tune of Peter, Paul and Mary's "Leaving on a jet plane." I leave it to the reader to fill in the blanks.

Eventually, using high-frequency jet ventilation (HFJV), we saved a baby that was not expected to survive. This progressed to using the jet more frequently and earlier in a baby's course. Today we selectively use the jet as the first intention for a list of pathologies and antenatal histories. Again, our CLD rates remained low and actually improved.

Today, our practice offers HFO and/or HFJV to babies as the first intention, and very few babies are managed with CV. For example, our 2016 ventilation hours were 14664 hours of HFO, 22584 hours of HFJV, and only 816 hours of CV. These numbers hardly represent HFV use as a rescue. Most notably, our CLD rate in 2015 was $8.1 \%$.

"The history of HFV is full of

controversy. Early studies, notably the dreadful "HIFI" study of the early '80s, did not favour HFV; indeed, quite the opposite was the case. Later analysis suggested the problem was not in the mode, but rather the practice: mean airway pressures were too low, and an "open lung" strategy was not employed."

The history of HFV is full of controversy. Early studies, notably the dreadful "HIFI" study of the early '80s, did not favour HFV; indeed, quite the opposite was the case. Later analysis suggested the problem was not in the mode, but rather the practice: mean airway pressures were too low, and an "open lung" strategy was not employed. When the "HIFI" results were excluded from metaanalysis, outcomes were similar between HFO and CV.(1)

Attending the annual "Snowbird" conference on high-frequency ventilation of infants and children was eye-opening. Studies were presented that showed that, at the very least, HFV was both safe and effective, at least as much as CV. Many didn't reach statistical significance, but all came close. A large study by Durand et al. out of Oakland Children's was the first I saw to establish the safety of HFO (2) definitively. Later studies confirmed the relative safety of HFO. (3) More recent studies suggest better neurodevelopemental outcomes with HFO than CV(4) and better pulmonary

NEONATOLOGY TODAY is interested in publishing manuscripts from Neonatologists, Fellows, NNPs and those involved in caring for neonates on case studies, research results, hospital news, meeting announcements, and other pertinent topics.

Please submit your manuscript to: LomaLindaPublishingCompany@gmail.com 
outcomes at age 11-14 in babies managed with HFO c.f. CV.(5)

Many of the more studies favouring HFO are from Great Britain or Europe where they use 2(nd) and 3(rd) generation machines such as the SLE and SLE $6000 \AA$, a ventilator with a piston-driven HFO option, the now obsolete Drager Babylog $®($ )8000 plus, and the Drager $\mathrm{VN}-500 \AA$ which also gives the option of volume targeted HFO. The latter two machines are pneumatically driven oscillators. American investigators have been limited to using the Sensormedics $3100 a \circledR$. One cannot compare the latter machine, a first-generation stand-alone oscillator, with the second and thirdgeneration multi-function ventilators. It is speculative; however, the difference in the devices may account for more favourable outcomes with newer, more sophisticated devices. Certainly, the ability to monitor and target HFO volumes should facilitate gentler, more lung-protective ventilation. These newer machines also provide the option of providing recruitment breaths to reverse atelectasis as they incorporate a CV ventilator.

\section{"Certainly, the ability to monitor and target HFO volumes should facilitate gentler, more lung-protective ventilation. These newer machines also provide the option of providing recruitment breaths to reverse atelectasis as they
incorporate a CV ventilator."}

An early study comparing HFJV with $\mathrm{CV}$ in the early treatment of respiratory distress syndrome showed a significant increase in adverse outcomes, namely cystic periventricular leukomalacia (PVL). It showed no correlation between hypocarbia and PVL.(6 ) There was no mention of the rapidity of changes in $\mathrm{PaCO}_{2}$ which, in my mind, are more deleterious than hypocarbia itself. A study by Kezler also showed significantly better outcomes with HVJV than CV.(6 ) It is important to note the Kezler study utilized an open lung strategy and showed no difference in the incidence of PVL or IVH with HFJV. When combined with the HIFI trial outcomes, it is clear that an open lung approach to ventilation is more important when bleeds are concerned than the mode of ventilation itself. The use of HFV was still in its infancy when these trials were conducted, and I suspect variations in practice and strategy were responsible for adverse outcomes and not the modes themselves. As clinicians increasingly understand the importance of open-lung ventilation, it is my opinion; the evidence will continue to support HFV's safety.

In addition to published data, experience in the unit in which I practice does not support an increased risk of brain bleeds or PVL using HFO or HJFV; our numbers are in line with our comparators. Given the large number of micro-preemies seen in our unit, I can say this with confidence.

It is unlikely further studies on HFJV will be done if such studies withhold the modality. Those who use the jet well have little or no equipoise and are unlikely to participate, although studies comparing HFO with HFJV would be useful; a Cochrane search for said modalities will come up empty. (7)

Several units with very good outcomes use HFV as the first intention for those babies requiring invasive support, my own included. While not empirical evidence, the sum of the parts surely gives a picture of the whole.

Given the current state of evidence, I submit it is high time to stop considering HFV as rescue therapy and utilize it more often and earlier in a baby's clinical course. When doing so, the importance of using adequate mean airway pressure/PEEP cannot be understated. We need to stop placing infants in a position wherein they require "rescue."

References:

1. https://pediatrics.aappublications.org/ content/98/6/1058?download=true

2. https://pubmed.ncbi.nlm.nih.gov/12200551/

3. https://www.ncbi.n/m.nih.gov/pmc/articles/PMC2672829/

4. https://pubmed.ncbi.nlm.nih.gov/17339385/

5. https://www.ncbi.n/m.nih.gov/books/NBK262116/

6. $\quad$ https://www.ncbi.n/m.nih.gov/pmc/articles/PMC7043304/

7. https://www.ncbi.n/m.nih.gov/pmc/articles/PMC6769183/

Disclosures: The author receives compensation from Bunnell Inc for teaching and training users of the LifePulse HFJV in Canada. He is not involved in sales or marketing of the device nor does he receive more than per diem compensation. Also, while the author practices within Sunnybrook H.S.C. this paper should not be construed as Sunnybrook policy per se. This article contains elements considered "off label" as well as maneuvers, which may sometimes be very effective but come with inherent risks. As with any therapy, the riskbenefit ratio must be carefully considered before they are initiated.

NT
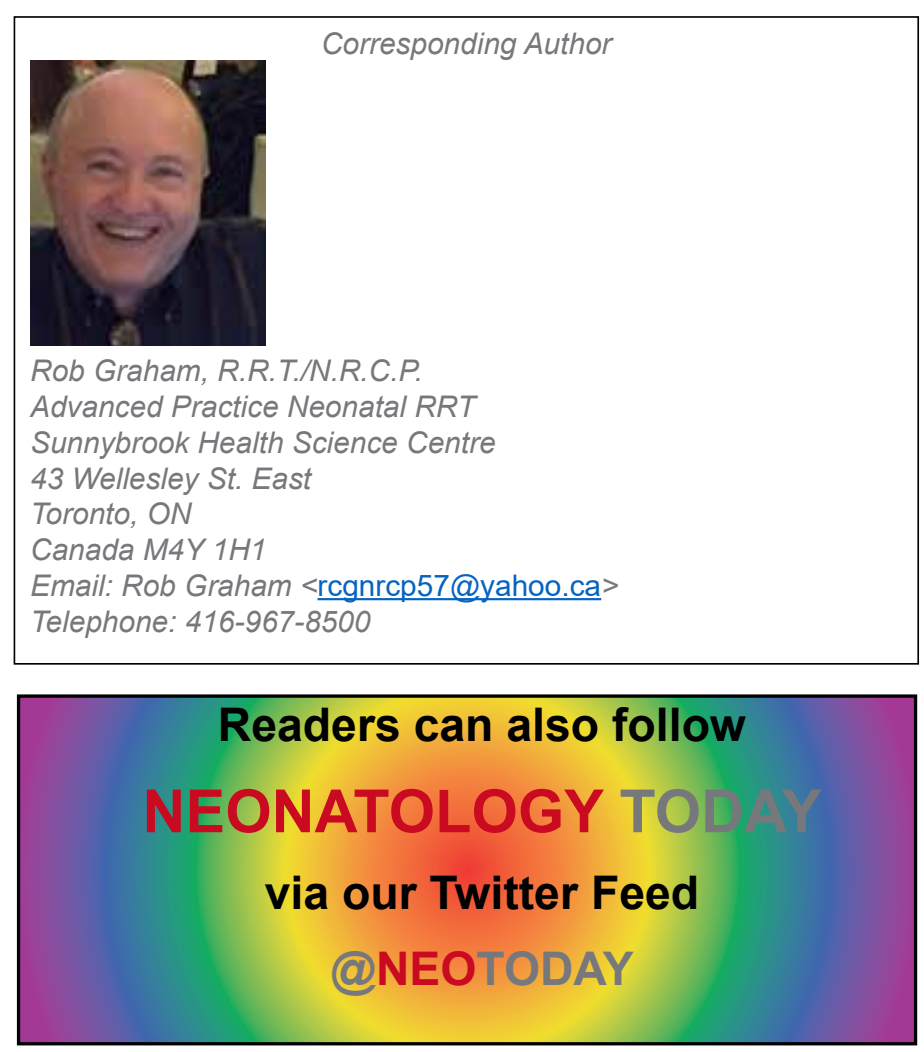